\title{
COMPARISON OF TEMPORAL REALISTIC
}

\section{TELECOMMUNICATION BASE STATION}

\author{
EXPOSURE WITH WORST-CASE
}

\section{ESTIMATION IN TWO COUNTRIES}

Zaher Mahfouz $^{*}$, Leen Verloock ${ }^{* *}$, Wout Joseph ${ }^{* *}$, Emmeric Tanghe $^{* *}$, Azeddine Gati*, Joe Wiart ${ }^{*}$, David Lautru $^{* * *}$, Victor Fouad Hanna ${ }^{* * *}$, and Luc Martens ${ }^{* *}$

* Orange Labs, France Télécom Division R\&D, RESA/WASA 38-40, rue Général Leclerc 92794 Issy Les Moulineaux, France

**Department of Information Technology, Ghent University / iMinds Gaston Crommenlaan 8, B9050 Ghent, Belgium

${ }^{* * *}$ UPMC Univ Paris 06, UR2, L2E, F-75005, Paris, France (email:wout.joseph@intec.UGent.be, fax:+32 933 14899)

Running title: Temporal realistic exposure

Acknowledgement: W. Joseph is a Post-Doctoral Fellow of the FWO-V (Research Foundation-Flanders). 
Abstract- The influence of temporal daily exposure to Global System for Mobile Communications (GSM) and Universal Mobile Telecommunications Systems (UMTSHSDPA) is investigated using spectrum analyzer measurements in two countries, France and Belgium. Temporal variations and traffic distributions are investigated. Three different methods to estimate maximal electric field exposure are compared. The maximal realistic $(99 \%)$ and the maximal theoretical extrapolation factor used to extrapolate the measured Broadcast Control Channel (BCCH) for GSM and the Common Pilot Channel (CPICH) for UMTS are presented and compared for the first time in the two countries. Similar conclusions are found in the two countries for both urban and rural areas: worst-case exposure assessment overestimates realistic maximal exposure up to $5.7 \mathrm{~dB}$ for the considered example. In France, the values are the highest, because of the higher population density. The results for the maximal realistic extrapolation factor at the weekdays are similar to those from weekend days.

Key Words- temporal exposure, electromagnetic fields, radio frequency, general public.

\section{INTRODUCTION}

People are continuously exposed to radio frequency (RF) electromagnetic fields emitted by wireless communication systems and broadcasting systems (both television and radio). Human exposure has to be assessed in accordance with international guidelines. This evaluation cannot be limited to a single measurement moment and should account for temporal variations of RF electromagnetic field exposure. The International Commission on Non Ionizing Radiation Protection ${ }^{(1)}$ and the Institute of Electronics and Electrical Engineers 
$\mathrm{IEEE}^{(2)}$ recommended exposure guidelines. Standards have also been developed to check the compliance with limits like those from the European Committee for Electro-technical Standardization CENELEC ${ }^{(3)}$ and the International Electrotechnical Commission (IEC) and form $\operatorname{IEEE}^{(2)}$.

The CENELEC method proposes to check the compliance to the reference levels, by extrapolating an instantaneous measurement of the pilot signals strength to the "worst-case" values. The worst-case assessment is adopted by some countries to ensure reliable and reproducible exposure assessments in varying traffic conditions, which guarantees the compliance. However, the extrapolation to maximum traffic does not consider the actual reallive exposure induced by the fluctuations of the field strength during communication.

Temporal variations and measurement procedures for temporal exposure assessment of RF signals are investigated in refs $(3,4,5,6,7,8)$. In ref (7), the daily distribution of the RF field strength is determined for FM Broadcasting (FM), Television (TV), Global System for Mobile Communications (GSM900 at $900 \mathrm{MHz}$ and GSM1800 at $1800 \mathrm{MHz}$ ), Universal Mobile Telecommunications Systems (UMTS), and High Speed Downlink Packet Access (HSDPA) services. The proposed method gives to the different signals a conservative extrapolation factor to determine the maximum real exposure during a day using a statistical method on different measurements performed over $24 \mathrm{~h}$. Erlang data, representing average mobile phone traffic intensity during a period of time, is related to RF exposure using temporal measurements during a week in ref (6). Diurnal variations of fields of mobile telecommunication and broadcasting systems are studied in Greece (5). Data from surveys of radio base stations in 23 countries from the year 2000 onward is pooled to investigate 
chronological trends and compare exposures by technology ${ }^{(8)}$. Daily variations are not accounted for in ref (8).

The objectives and novelties in this paper are the following. Firstly, we estimate the maximum exposure in two countries (Belgium and France) in urban and rural zones by comparing the extrapolation methods of ${ }^{(6,7)}$ and CENELEC ${ }^{(3)}$ for the first time. Secondly, we determine the maximal realistic (99\%) and the maximal theoretical extrapolation factor used to extrapolate the measured Broadcast Control Channel (BCCH) for GSM and the Common Pilot Channel (CPICH) for UMTS/HSDPA and the comparison in two countries. Thirdly, for the determination of the realistic extrapolation factors, we distinguish here between GSM900, GSM1800, UMTS, and HSDPA, and two types of environments (urban and rural), and weekdays versus weekend days, which is novel. Fourthly, the methods to assess the electric field exposure at a time instant from the electric field measurements at another time instant, and to estimate the maximum electric field value over a day are compared. The first method (Erlang method ${ }^{(6)}$ ) is based on the Erlang data for GSM signals and on the throughput data or transmitted power for the UMTS signals. The second method (3-Gaussian $\operatorname{method}^{(7)}$ ) is based on a statistical analysis using a three-Gaussian distribution model. The third method is the one of CENELEC ${ }^{(3)}$ to extrapolate to maximal field values.

\section{MATERIALS AND METHOD}

\section{A. Configurations and measurement setups}

In Belgium, the electric fields are measured with a tri-axial R\&S TS-EMF Isotropic Antenna (dynamic range of $1 \mathrm{mV} \mathrm{m}^{-1}-100 \mathrm{~V} \mathrm{~m}^{-1}$ and a frequency range of $30 \mathrm{MHz}-3 \mathrm{GHz}$ ) 
in combination with a spectrum analyzer SA of type R\&S FSL6 (frequency range of $9 \mathrm{kHz}-$ $6 \mathrm{GHz}$ - http://www2.rohde-schwarz.com/). To demodulate the UMTS and HSDPA signals, the FSL-K72 decoder option of the R\&S SA is used. Using this option, the code domain analyzer can be used for demodulation of the UMTS signals. The tri-axial antenna has a builtin switch to select the appropriate axis during the measurements.

In France, the measurement equipment consisted also of a spectrum analyzer (Agilent MXA, N9020A model, frequency band: 20Hz-13.6 GHz, www.agilent.com), an isotropic tri-axial probe satisfying the specifications of the EN50492 standard $^{(3)}$, a switch that selects the axis of the probe to the measured unit, and a laptop controller to control the analyzer and the switch, and to save all the measurements. For UMTS (3GPP R99) and HSDPA systems, a WidebandCode Division Multiple Access (W-CDMA) decoder (Anritsu, ML8720B model, www.anritsu.com) is used for measurements to extract the total power and also the allocated power to each channel which is not possible with a standard spectrum analyzer.

The measurement systems in both studies are thus very similar: these consist of narrowband equipment i.e., a spectrum analyzer and suitable measurement probes. In both studies also a W-CDMA decoder is used to extract the power allocated to each channel. These measurement setups have been validated extensively in previous studies ${ }^{(4,6,7)}$ and satisfy the CENELEC standard $^{(3)}$, thus they are comparable. The main difference between both studies is that in Belgium, we had collaboration of the mobile operators, so the contribution per operator could be determined. The measurement uncertainty for the electric field is $\pm 3 \mathrm{~dB}$ for the considered SA setup ${ }^{(3)}$. This uncertainty represents the expanded uncertainty evaluated using a confidence interval of $95 \%$. 


\section{B. Measurement procedure}

Fig. 1 summarizes the measurement procedure for the temporal assessment of RF base station exposure in Belgium and France.

In Belgium, 16 sites were considered, 7 sites in rural environments and 9 in urban environments (11 outdoor sites and 5 indoor sites). The sites were investigated during 2 different measurement campaigns. A first measurement campaign was performed in Brussels, Belgium; long-term temporal measurements were performed here during 7 days for 2 sites in rural zones and 3 sites in urban zones. Additionally, in Flanders, Belgium, 5 sites in rural zones and 6 sites in urban zones were investigated during 2 weekdays and 2 working days.

For all the sites, a spectral overview measurement (step 1 in Fig. 1) in the frequency range of $80 \mathrm{MHz}$ up to $3 \mathrm{GHz}$ has been performed to identify present RF signals. Based on this spectral overview the relevant present signals (FM, GSM900, GSM1800, HSDPA, UMTS and demodulation of the UMTS-HSDPA signal for the different operators in Belgium) were selected for the long-term measurements (step 2). These significant signals were measured sequentially for each of the three orthogonal components and each measurement sequence was repeated during several days (step 3). Depending on the number of significant signals, a measurement sequence took about 2-4 minutes. Performing these measurements during 7 and 4 days resulted for each significant signal in a total of 2,520 and 1,440 samples, respectively. The total number of time samples was 115,967 for urban zones and 100,419 for rural zones. In Belgium, three different operators are active for commercial GSM and UMTS networks. For the measurements and the statistical analysis, the signals for the different operators were processed separately for each kind of signal (e.g., GSM900, GSM1800 ...). 
In Paris, France, 6 measurements at 3 different sites in rural zones and 22 measurements in 9 different sites in urban zones were performed. Also, first a spectral measurement (step 1 in Fig. 1) was performed to identify present RF signals (step 2). Measurements were performed with a sampling rate of 10 s for each site in different locations (bedroom, kitchen, living-room) to obtain a wide range of situations (step 3). Each of these measurements resulted in a total of 8,570 measured samples per signal during one day. The total number of time samples was 51,420 per signal for rural zones and 188,540 per signal for urban zones. For UMTS and HSDPA systems, 8 measurements for UMTS and 12 measurements for HSDPA were performed with a sampling rate of $6 s$ in 5 different sites in urban areas. For each measurement a total of 15,000 measured samples was obtained during one day.

Different factors can influence the RF measurements (path loss, the data rate, discontinuous transmission (DTX), power control...). This has been addressed in previous publications by making network adaptations to enable that research ${ }^{(9,10)}$. Note that in this paper, the measurements were performed continuously during 24 hours to take into account all these aspects. The networks were not forced to operate in a specific mode in order to observe traffic variations in real conditions. From the obtained data the maximal exposure factors can be assessed (Fig. 1).

\section{Method to assess realistic maximal extrapolation factors}

The 3-Gaussian method ${ }^{(7)}$ is compared with the CENELEC extrapolation method ${ }^{(3)}$ to estimate the maximum exposure in two countries (Belgium and France). Both methods are based on the determination of the extrapolation factor to use in combination with the pilot signals. Basically, GSM and UMTS-HSDPA are signals composed of two parts. The first part 
consists of the pilot signals. The second part consists of the traffic signals. BCCH and the $\mathrm{CPICH}$ are the pilot signals for GSM and UMTS-HSDPA respectively. BCCH and CPICH represent the signalizations and they are continuously emitted over the day.

To extract the broadcast control channel BCCH for GSM using spectral measurements the following method is used. Spectral scans in the appropriate GSM frequency band enable to identify the different GSM channels. The BCCH signals can be identified because these never become zero, in contrary to the TRX signals that vary largely between zero (no phone traffic) and maximal field values (lots of traffic) ${ }^{(4)}$. Moreover, for hopping (i.e., changing the channel frequency with time) the frequencies of the hopping channels are known or provided by the mobile operators. Fig. 2 shows as an example the frequency spectrum (hopping) for a GSM900 signal measured during 24 hours in Belgium. Fig. 2 shows 396 traces of momentary measurement values of the electric field captured with the SA: clearly 4 BCCH channels and various traffic (TRX) channels (vary from noise level to maximal values) can be separated in the downlink band. For UMTS-HSDPA, the measurements occurred with a code domain analyzer (see Section measurement setup) where the CPICH and traffic channels are extracted. Again, BCCH and $\mathrm{CPICH}$ signals never become zero in contrary to the electricfield strength of the TRXs ${ }^{(4)}$.

In theory, the $\mathrm{BCCH}$ and $\mathrm{CPICH}$ power remains constant but in-situ the electric field strengths of the BCCH and $\mathrm{CPICH}$ vary over time due to environmental changes (e.g., movement of people and cars, weather) and instability of the $\mathrm{BCCH}^{(6)}$. The traffic variation is represented by the ratio $\left(\mathrm{N}_{\text {traffic }}(\mathrm{t})\right)$ of the total power $\left(\mathrm{P}_{\text {total }}(\mathrm{t})\right)$ to the pilot power $\left(\mathrm{P}_{\text {pilot }}\right)$ as follows: 


$$
N_{\text {traffic }}(t)=\frac{P_{\text {total }}(t)}{P_{\text {pilot }}}
$$

With t representing time (s).

Some standards ${ }^{(3,12)}$ propose an extrapolation method to assess the maximal "worst-case" electric field, based on the measurements of the pilots signals. Network operators can provide the maximal theoretical value of the traffic channels. Extrapolation using these numbers often overestimates the realistic electric-field values because traffic channels vary during the day and these numbers represent the maximal capacity of the cell. For control agencies, it is important to extrapolate using realistic extrapolation factors to check compliance with limits. In this paper, $\mathrm{N}_{\text {TRX }}$ and $\mathrm{N}_{\text {CPICH }}$ are the equivalent traffic ( $\mathrm{N}_{\text {traffic }}$ in (2)) evaluated for GSM and UMTS, respectively. Note that the maximum numbers of traffic (TRXs) channels (and CPICHs) are set by telecommunication operators through radio planning optimization taking into account the population density and the traffic capacity. Systems are also designed to avoid a maximum load to allow a good traffic quality.

In France, the maximum number of TRXs has been defined by national authorities as a constant value depending on the population density and in practice this represents $99 \%$ of the actual installed cases. The values of the maximum deployed TRXs by operators for GSM900 and GSM1800 networks are 6 and 8, respectively. These maximum values have been recommended by the National Agency of Frequency (ANFR) ${ }^{(11)}$ to assess the exposure for maximum traffic situation (worst case). $\mathrm{In}^{(13,14)}$, the value of 10 is recommended for $\mathrm{N}_{\mathrm{CPICH}}$ in UMTS network, which corresponds to the maximum theoretical value (recommended also by ANFR). There is no value recommended yet for $\mathrm{N}_{\mathrm{CPICH}}$ in HSDPA systems. 
In Belgium, the different regional regulations do not include any attempt to extrapolate to the maximum exposure. The electric-field values averaged over 6 minutes have to be compliant with regional limits at each moment of the day, but no further details are included.

In this study, the determination of the real number of equivalent TRXs and CPICHs ( $\mathrm{N}_{\text {TRX }}$ and $\mathrm{N}_{\mathrm{CPICH}}$ ) that is active over one day in France and in Belgium using the method proposed in ${ }^{(7)}$ is presented. To find the realistic maximum number of TRX-CPICH values, the $99 \%$ value is taken for each measured signal. For the measurements in Belgium, the 99-percentile value of the TRX-CPICH is evaluated for each site and for each operator. This is not the case for the measurements in France, where only one model and one TRX-CPICH value (max at 99\%) is available for each technology because all the measurements for one technology were processed together.

\section{Summary of existing methods for extrapolation self}

The CENELEC method ${ }^{(3)}$ proposes to check the compliance to the reference levels, by extrapolating an instantaneous measurement of electric field strength of the pilot signals (BCCH and $\mathrm{CPICH}$ ) to the "worst-case" value. This method does not allow determining the electric field exposure at a time instant from the electric field measurements at another time instant. Therefore, new methods are needed to evaluate the real exposure.

$\mathrm{In}^{(4,6)}$, a method is proposed to calculate the maximal electric field strength during 24 hours (1 day) from the momentary total measured electric field value (pilot signals and traffic signals together) using the corresponding Erlang data for GSM. The maximal electric field strength ( $E_{\text {calc }}^{\max }$ ) can be calculated from a momentary field value $E_{\text {norm }}(\mathrm{t})$ using eq. (2). 


$$
E_{\text {calc }}^{\max }=E_{\text {mom }}(t) \frac{\max (\text { Erlang })}{\operatorname{Erlang}(t)}=E_{\text {mom }}(t) S F_{\text {Erlang }}(t) .(V / m)
$$

With Erlang(t) and max(Erlang) are the Erlang data at time instant $t$ and the maximum Erlang data during $24 \mathrm{~h}$ for the corresponding electric field value of the GSM signal at a specific location, respectively. $S F_{\text {Erlang }}$ is the normalized Erlang data as function of the time. Fig. 3 shows the median value of all the normalized Erlang data as function of the time between 9:00 am and 6:00 pm for the different considered sites provided by the telecom operators (15 data sets for GSM 900 and GSM1800, Section II.A). The chosen time interval corresponds to typical working hours during which control agencies can make their measurements. One has to remark that the traffic data must be provided by the operator. In this paper, this method is applied for GSM because only the Erlang data was provided. For UMTS/HSDPA no traffic data was available.

$\mathrm{In}^{(7)}$, the method proposes to obtain the $\mathrm{N}_{\mathrm{TRX}}(\mathrm{t})$ over 24 hours. Based on the explanation of Section II.C, this factor is found with a statistical method on different measurements performed at different sites. This factor can be used whatever the location of the performed measurements. The scaling factor $S F_{3-\text { Gaussian }}$ is defined as follows:

$$
S F_{3-\text { Gaussian }}=\frac{E_{\max }}{E(t)}(-)
$$

(-) representing no unit. Fig. 3 shows also the scaling factor using eq. (3) obtained using the data presented in this paper. In the following, $\mathrm{N}_{\mathrm{TRX}}$ and $\mathrm{N}_{\mathrm{CPICH}}$ will be determined for realistic circumstances in the two countries. 


\section{RESULTS AND DISCUSSION}

\section{A. Comparison of realistic maximal exposure}

A summary of the median and maximum values of the realistic extrapolation factors $\left(\mathrm{N}_{\text {traffic }}\right.$ in eq. (1), 99-percentiles) for the different technologies is provided in Table 1 . In this table, the theoretical maximum values for France are those recommend by ANFR; the theoretical maximal values for the measured sites were not available. For the measurements in Belgium, the theoretical maximum values are these provided by the network operators for the corresponding sites. The 99-percentile values of $\mathrm{N}_{\mathrm{TRX}}$ and $\mathrm{N}_{\mathrm{CPICH}}$ are also evaluated by taking a sliding time average of 6 min for all the measurements in agreement with the ICNIRP exposure guidelines $^{(1)}$.

Fig. 4 shows the cumulative distribution function (CDF) of the measured realistic values of the $99^{\text {th }}$ percentile of $\mathrm{N}_{\mathrm{TRX}}$ and $\mathrm{N}_{\mathrm{CPICH}}$ for the GSM900, GSM1800, UMTS, and HSDPA technologies in Belgium. Clearly, all realistic values are lower than the theoretical maxima of Table 1 for the various technologies. The realistic values in Table 1 are obtained by taking the maximum and the median values of the CDFs of these realistic 99-percentile values in Fig. 4.

In France and in Belgium, the results of Table 1 show that the realistic maximal $\mathrm{N}_{\mathrm{TRX}}$ in urban zones for GSM1800 (4.0 in France and 4.3 in Belgium, no averaging) is found higher than for GSM900 (3 in France and 4 in Belgium). This result was expected because the GSM1800 network is used as a reinforcement network to increase the GSM900 capacity. Therefore, $\mathrm{N}_{\text {TRX }}$ in a GSM1800 network is larger than in a GSM900 network in urban zones. This is not the case in rural zones, because GSM900 (e.g., in Table 1 maxima of 4.0 and 3.4 for GSM900 
versus 3.0 and 2.7 for GSM1800, in France and Belgium, respectively) is preferred due to the propagation performances of the $900 \mathrm{MHz}$ carrier in this environment and also because GSM900 was deployed first and no more capacity is needed in rural environments. This explains why the maximal $\mathrm{N}_{\text {TRX }}$ found for GSM1800 is smaller in rural than in urban zones. Also for the theoretical maxima these conclusions can be drawn.

Operators in France and in Belgium use UMTS most of the time for voice communications while HSDPA is used for data. UMTS and HSDPA are very similar in terms of signal characteristics and radio access interface. However, they are different in terms of power allocation. In fact, in HSDPA systems, all the power is allocated to one user equipment at a time, which explains why the $\mathrm{N}_{\mathrm{CPICH}}$ for HSDPA is higher than UMTS in Table 1 . Moreover, the theoretical values for HSDPA are higher in Belgium than in France, explaining the highest realistic values in Belgium for HSDPA.

The values for UMTS are the highest in France, because of the higher population and also because of the network optimization. Note that in Belgium, the UMTS network is not yet deployed as a GSM network, which is the reason for higher realistic values for GSM900GSM1800 in urban areas in Belgium than in France.

Similar conclusions can be made in the two countries for both urban and rural areas: the maximal equivalent TRX and CPICH values in a realistic environment demonstrate that a full traffic situation is not reached in reality. 


\section{B. Weekdays versus weekend days}

In Belgium, for each site, measurements were performed on weekdays and weekend days. Table 2 lists their median values of the $99 \%$ value for $\mathrm{N}_{\mathrm{TRX}} / \mathrm{N}_{\mathrm{CPICH}}$. Fig. 5 compares the CDFs for GSM900 in urban zones.

The median values are very similar for all types of technology in an urban environment for both weekdays and weekend days, in general only slightly higher values are obtained for weekdays (see also Fig. 5 for GSM900). Thus, realistic maximal traffic in urban environments does not depend much on the day of the week. In rural environments (Table 2), the $\mathrm{N}_{\mathrm{CPICH}}$ values for HSDPA are higher on weekdays (4.9) than on weekdays (3.8), indicating that here more data traffic is present on weekdays.

\section{Comparison of the Maximal Daily Electric Field Value Using Different Methods}

In this section, three methods are presented and compared to find the realistic scaling factors, in order to extrapolate an instantaneous measurement to the maximal value possible over a day.

\section{1) Comparison of the Erlang and 3-Gaussian methods}

The scaling factors of Fig. 3 for both methods as function of the time show a similar trend (for GSM900 and GSM1800). Lowest scaling factors (corresponding to highest measured instantaneous electric field values) can be found between noon and evening. Then, the scaling factors are for both methods lower than 1.4. Between 9:00 am and noon both methods have a decreasing scaling factor but the scaling factor based on the Erlang data gives higher values. The scaling factor based on the Erlang data is only considered during normal working hours while the scaling factor based on the 3-Gaussian model is given for the whole day. One has to 
remark that the scaling factors to extrapolate instantaneous measurements to the maximum possible value over the day depend on the traffic pattern of the mobile phone users and can change over time. Consequently, the values must be evaluated at regular time intervals.

2) Extrapolation for an instantaneous measurement with the different proposed methods

In Table 3, a comparison of the different methods to calculate the maximum electric-field value can be found for a realistic long-term measurement, an example of a GSM signal, performed in Belgium. Both the instantaneous electric-field value during 24 hours and the pilot signals were measured. $E_{\max }^{\text {true }}$ is the maximum measured electric-field value during 24 hours. The maximum calculated electric-field value $E_{\max }^{\text {calc }}$ is also assessed using the different methods proposed in this paper. The first method is based on the extrapolation method mentioned in CENELEC ${ }^{(3)}$. The scaling factor is determined in three different ways: (i) using the real number of traffic channels TRXs for the specific site provided by the operators, (ii) using the equivalent number of TRXs based on the 3-Gaussian method (see Table 1), and (iii) using the number of TRXs mentioned in ANFR. The BCCH is not constant over time ${ }^{(4)}$, so we have chosen the mean value of the BCCH over 24 hours to perform the extrapolation. The second and third method are based on the scaling factors of the Erlang data ${ }^{(6)}$ and of the 3Gaussian method ${ }^{(7)}$. The scaling factors $\left(\mathrm{SF}_{\text {Erlang }}\right.$ and $\left.\mathrm{SF}_{3-\text { Gaussian }}\right)$ are determined as a function of time to rescale the momentary total measured electric field value (measured at 11:00 am in our example). For the first method, a dedicated measurement equipment to measure the pilot signals is required while the total measured electric field, used for the second and third method, can be measured using a simple spectrum analyzer. The results are summarized in Table 3. In this table, the deviations $\Delta$ between the realistic maximum and the calculated maximum electric field are also listed: 


$$
\Delta=20 \log \frac{E_{\max }^{\text {calc }}}{E_{\max }^{\text {true }}}(\mathrm{dB})
$$

The results of Table 3 confirm that the CENELEC method ${ }^{(3)}$ overestimates the real exposure $(+1.7 \mathrm{~dB}$ to $5.7 \mathrm{~dB})$, which is a high value compared to the proposed methods. The results found with the maximal $\mathrm{N}_{\mathrm{TRX}}$ given from the operator $(0.27 \mathrm{~V} / \mathrm{m}$ corresponds to a full traffic situation as the maximal known $\mathrm{N}_{\mathrm{TRX}}$ is used at this location) confirm that a situation with a full traffic is not reached in reality at this given location as the maximal real value found over 24 hours is only about $0.21 \mathrm{~V} / \mathrm{m}$. For the CENELEC method, extrapolation using the 99percentile value (3-Gaussian method), gives lowest deviations (an overestimation of $1.7 \mathrm{~dB}$ ). The methods proposed in ${ }^{(4,6,7)}$ give a better assessment of the maximal electric field value from an instantaneous measurement (only $+0.4 \mathrm{~dB}$ to $+0.6 \mathrm{~dB}$ for this example).

Finally, Table 3 compares also the electric-field values of the different methods with the ICNIRP reference levels ${ }^{(1)}$. Therefore, the exposure ratio (ER in in \%) of a signal is defined as the ratio in \% of the obtained electric-field value to the corresponding ICNIRP reference level $^{(1)}$ for the general public. The percentages in Table 3 are much lower than $100 \%$, indicating compliance with the ICNIRP guidelines. The 3-Gaussian and Erlang methods give $\mathrm{ER}=0.5 \%$, which agrees very well with the true maximal ER value of $0.5 \%$. The CENELEC method gives ratios up to $1 \%$, thus an overestimation of a factor 2 is here possible. If exposure values would be close to the ICNIRP reference levels or regional limits, this overestimation can result in non-compliance while in reality compliance would be obtained.

\section{CONCLUSIONS}

In this paper, the influence of the temporal daily exposure to GSM, UMTS, and HSDPA is investigated. Temporal variations and traffic distributions are compared in two 
different countries, France and Belgium. The electric-field amplitude varies because it depends on the environmental changes and the traffic.

The number of actual traffic channels $\mathrm{N}_{\mathrm{TRX}}$ and $\mathrm{N}_{\mathrm{CPICH}}$ evaluated for GSM and UMTS, respectively, are determined in the two countries. Similar conclusions are found in the two countries for both urban and rural areas. The maximal $\mathrm{N}_{\mathrm{TRX}}$ in GSM1800 is higher than the one of GSM900 in urban zones. This is not the case in rural zones, because GSM900 is preferred due to the propagation performances of the $900 \mathrm{MHz}$ carrier in this environment. The maximal equivalent TRX and CPICH values found in a realistic environment demonstrate also that a full traffic situation is never reached in reality, and that the CENELEC method (worst-case assessment) overestimates the real exposure.

The Erlang and 3-Gaussian methods to assess the electric field exposure at a time instant from the electric-field measurements at another time instant, and to estimate the maximum electricfield value over a day from an instantaneous measurement are compared. These methods are also compared with the CENELEC method. Again, the CENELEC method overestimates the real exposure much more compared to the proposed methods.

Future research should focus on the temporal evolutions of LTE (long term evolution), which is recently launched in several countries.

\section{REFERENCES}

1. International Commission on Non-ionizing Radiation Protection (ICNIRP). Guidelines for limiting exposure to time-varying electric, magnetic, and electromagnetic fields (up to 300 GHz). Health Phys 74(4), 494-522 (1998). 
2. IEEE, Institute of Electrical and Electronics Engineers. IEEE standard for safety levels with respect to human exposure to radio frequency electromagnetic fields, $3 \mathrm{kHz}$ to $300 \mathrm{GHz}$. New York, USA. C95.1-2005 (2005).

3. CENELEC, European Committee for Electrotechnical Standardization. TC 106x WG1 EN 50492 in situ. Basic standard for the in-situ measurement of electromagnetic field strength related to human exposure in the vicinity of base stations. Brussels, Belgium: European Committee for Electrotechnical Standardization (2008).

4. Joseph, W., Verloock, L., Tanghe, E., Martens, L. In-situ measurement procedures for temporal RF electromagnetic field exposure of the general public. Health Phys 96(5), 529-542 (2009).

5. Manassas, A., Boursianis, A., Samaras, T., Sahalos, J. N. Continuous electromagnetic radiation monitoring in the environment: analysis of the results in Greece. Radiat Prot Dosimetry. 151(3), 437-42 (2012).

6. Joseph, W., Verloock, L. Influence of mobile phone traffic on general public base station exposure. Health Physics 99(5), 631-638 (2010).

7. Mahfouz, Z., Gati, A., Lautru, D., Wong, M-F., Wiart, J., Hanna, VF. Influence of Traffic Variations on Exposure to Wireless Signals in Realistic Environments, Bioelectromagnetics 33(4), 288-297 (2012).

8. Rowley, JT. and Joyner KH. Comparative international analysis of radiofrequency exposure surveys of mobile communication radio base stations. Journal of Exposure Science and Environmental Epidemiology 22(3): 304-315 (2012). 
9. Wiart, J., Dale, C., Bosisio, A.V., Le Cornec A. Analysis of the influence of the power control and discontinuous transmission on RF exposure with GSM mobile phones. IEEE Trans Electromagn Compat. 42(4), 376-384 (2000).

10. Gati, A., Hadjem, A., Wong, M.F., Wiart, J. Exposure Induced by WCDMA Mobiles Phones in Operating. IEEE Transactions on Networks 8(12), 5723-5727 (2009).

11. ANFR, National Agency of Frequency. (Agence Nationale de Fréquence en France) http://www.anfr.fr/fileadmin/mediatheque/documents/expace/protocole\%20de\%20mesure \%20DR15-2_1.pdf. (2004)

12. ECC, Electronic Communications Committee within the European Conference of Postal and Telecommunications Administrations (CEPT). ECC recommendation (02)04 (revised Bratislava 2003, Helsinki 2007) Measuring non-ionising electromagnetic radiation (9 kHz - $300 \mathrm{GHz})$, www.ero.dk (2004).

13. 3rd Generation Partnership Project (3GPP). Technical Specification Group Radio Access Network. Radio transmission and reception. (3GPP TS 05.05 V8.20.0). Sophia-Antipolis Cedex France: 3GPP (2005).

14. 3rd Generation Partnership Project (3GPP). Technical Specification Group Radio Access Network "Requirements for support of radio resource management (3GPP TS 25.133

V5.17.0). Sophia-Antipolis Cedex France: 3GPP (2005). 


\section{Author affiliations}

Zaher Mahfouz ${ }^{*}$, Azeddine Gati ${ }^{*}$, and Joe Wiart ${ }^{*}$

*Orange Labs, France Télécom Division R\&D, RESA/WASA 38-40, rue Général Leclerc 92794 Issy Les Moulineaux, France

Leen, Verloock**, Wout, Joseph**, Emmeric Tanghe**, and Luc Martens**

**Department of Information Technology, Ghent University / IBBT

Gaston Crommenlaan 8, B-9050 Ghent, Belgium, fax: +32 93314899

(email:wout.joseph@intec.UGent.be)

David Lautru $^{* * *}$, and Victor Fouad Hanna***

***UPMC Univ Paris 06, UR2, L2E, F-75005, Paris, France 


\section{List of captions}

Table 1: Median and maximal values for the number of equivalent TRX $\left(N_{T R X, 99 \%)}\right)$ and $C P I C H$ (N $\left.N_{C P I C H, 99 \%}\right)$ for GSM, UMTS, and HSDPA in urban and rural zones (in France and Belgium).

Table 2: Comparison of median values of equivalent TRX $\left(N_{T R X, 99 \%}\right)$ and $C P I C H\left(N_{C P I C H, 99 \%}\right)$ for GSM, UMTS and HSDPA on weekdays and weekend days (for the different operators at each site in urban and rural zones in Belgium).

Table 3: Real and calculated maximal electric field values determined with different extrapolation methods and deviation $\Delta$ between true and calculated value for a GSM900 signal (measurement performed in Belgium).

Figure 1: Block diagram of the measurement procedure for temporal RF base station exposure assessment.

Figure 2: Electric field (V/m) of BCCH and TRX channels of GSM900 signal (396 traces during 24 hours).

Figure 3: Scaling factor based on Erlang for GSM900 and GSM1800 signals (median for all sites during working hours) and based on the 3-Gaussian method for GSM900, GSM1800, UMTS, and HSDPA.

Figure 4: Cumulative distribution function (CDF) of the 99\% extrapolation factor for all sites in urban areas in Belgium (6 minutes averaging).

Figure 5: comparison of CDF of the 99\% extrapolation factor on weekdays and weekend days for all sites in urban areas for GSM900 (Belgium, 6 minutes averaging). 


\begin{tabular}{|c|c|c|c|c|c|c|c|c|}
\hline & & \multirow[b]{2}{*}{$\mathbf{N}_{\text {TRX,99\% }} / \mathbf{N}_{\text {CPICH,99\% }}$} & \multirow{2}{*}{$\begin{array}{l}\text { France } \\
\text { Urban } \\
\text { Max }\end{array}$} & \multirow{2}{*}{$\begin{array}{l}\text { Rural } \\
\text { Max }\end{array}$} & \multicolumn{2}{|c|}{$\begin{array}{l}\text { Belgium } \\
\text { Urban }\end{array}$} & \multicolumn{2}{|c|}{ Rural } \\
\hline & & & & & Max & Median & Max & Median \\
\hline $\mathbf{N}_{\text {TRX }}$ & Theoretical & & $6.0(*)$ & $6.0\left(^{*}\right)$ & 5.0 & 3.0 & 4.0 & 3.0 \\
\hline \multirow[t]{2}{*}{ GSM900 } & Realistic & No averaging & 3.0 & 4.0 & 4.0 & 2.4 & 3.4 & 2.5 \\
\hline & & 6 min. averaging & 2.36 & 3.2 & 4.0 & 2.4 & 3.4 & 2.1 \\
\hline $\mathbf{N}_{\text {TRX }}$ & Theoretical & & $8.0(*)$ & $8.0\left(^{*}\right)$ & 8.0 & 2.0 & 4.0 & 3.0 \\
\hline \multirow{2}{*}{ GSM1800 } & Realistic & No averaging & 4.0 & 3.0 & 4.3 & 2.6 & 2.7 & 2.2 \\
\hline & & 6 min. averaging & 2.7 & 2.2 & 4.3 & 2.5 & 2.3 & 2.0 \\
\hline $\mathbf{N}_{\text {CPICH }}$ & Theoretical & & $10(*)$ & - & 3.9 & 3.9 & - & - \\
\hline \multirow[t]{2}{*}{ UMTS2100 } & Realistic & No averaging & 5.0 & - & 2.3 & 2.1 & - & - \\
\hline & & $6 \mathrm{~min}$. averaging & 4.4 & - & 2.3 & 2.1 & - & - \\
\hline $\mathbf{N}_{\text {CPICH }}$ & Theoretical & & - & - & 20.0 & 14.8 & 20.0 & 15.0 \\
\hline \multirow{2}{*}{ HSDPA } & Realistic & No averaging & 14.0 & - & 18.7 & 7.9 & 9.8 & 5.4 \\
\hline & & $6 \mathrm{~min}$. averaging & 10.2 & - & 17.8 & 7.3 & 8.3 & 5.2 \\
\hline
\end{tabular}

$(\mathrm{x})^{*}=$ general value recommended by ANFR

Table 1 


\begin{tabular}{llll}
\hline $\mathbf{N}_{\mathrm{TRX}, 99 \%}$ & & Weekday & $\begin{array}{l}\text { Weekend } \\
\text { day }\end{array}$ \\
\hline Urban & GSM900 & 2.2 & 2.2 \\
& GSM1800 & 2.5 & 2.3 \\
& UMTS2100 & 2.1 & 2.0 \\
& HSDPA & 5.0 & 5.3 \\
Rural & GSM900 & 2.1 & 2.0 \\
& GSM1800 & 2.2 & 1.9 \\
& UMTS2100 & - & - \\
& HSDPA & 4.9 & 3.8 \\
\hline
\end{tabular}

Table 2 


\begin{tabular}{|c|c|c|c|c|}
\hline $\begin{array}{l}\text { Methods of calculation of the } \\
E_{\max } \text { for the measured site }\end{array}$ & & $\begin{array}{l}E_{\max } \\
(V / m)\end{array}$ & $\begin{array}{l}\Delta \\
(\mathrm{dB}) \\
\end{array}$ & $\begin{array}{l}\text { ER } \\
(\%) \\
\end{array}$ \\
\hline Max over $24 \mathrm{~h}$ & $E_{\max }^{\text {true }}$ & 0.210 & - & 0.5 \\
\hline \multirow{3}{*}{ CENELEC method } & $\begin{array}{l}E_{\max }^{\text {calc }} \text { based on BCCH and } \\
\mathrm{N}_{\text {TRX,realistic (given from the }} \\
\text { operator for this site) }\end{array}$ & 0.270 & 2.2 & 0.7 \\
\hline & $\begin{array}{l}E_{\max }^{\text {calc }} \text { based on } \mathrm{N}_{\mathrm{TRX}, 99 \%} \text { found } \\
\text { with the 3-Gaussian method }\end{array}$ & 0.250 & 1.7 & 0.6 \\
\hline & $\begin{array}{l}E_{\max }^{\text {calc }} \text { based on worst-case, } \\
\left(\mathrm{N}_{\mathrm{TRX}} \text { given from ANFR) }\right.\end{array}$ & 0.410 & 5.7 & 1.0 \\
\hline Erlang method & $E_{\max }$ based on $S F_{\text {Erlang, }}$ & 0.224 & 0.55 & 0.5 \\
\hline 3-Gaussian method & $E_{\max }$ based on $S F_{3 \text {-Gaussian }}$ & 0.221 & 0.44 & 0.5 \\
\hline
\end{tabular}

Table 3 


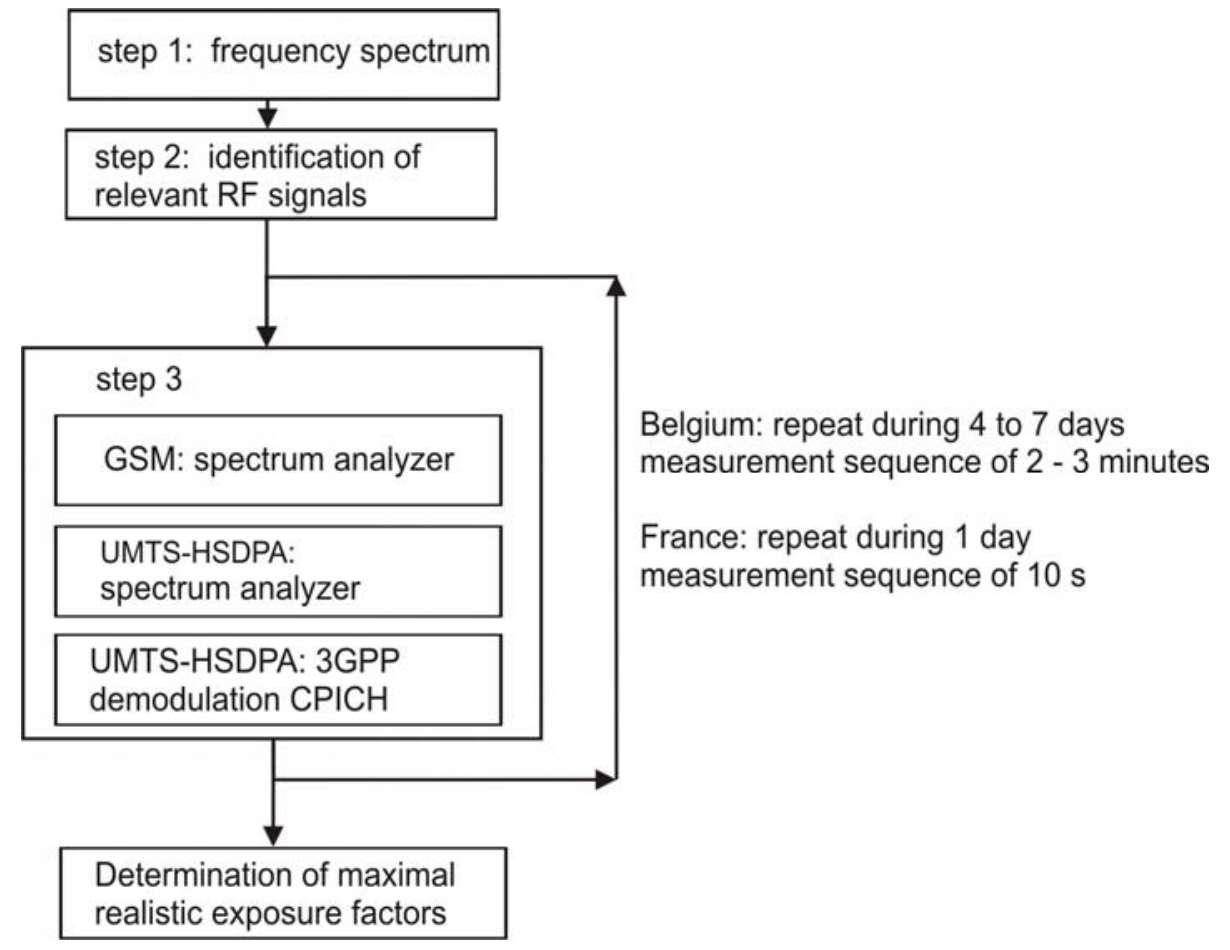

Figure 1 


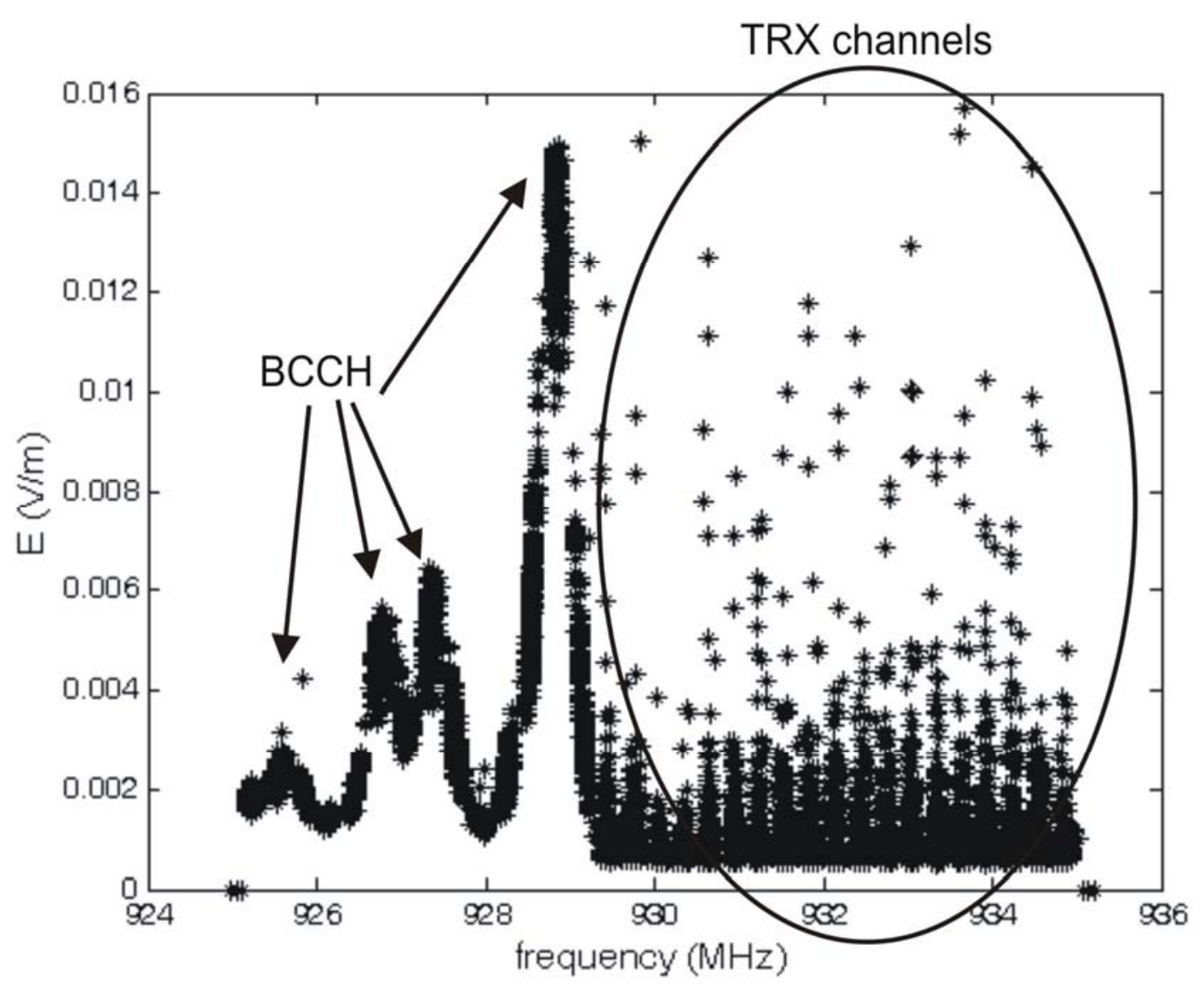

Figure 2 


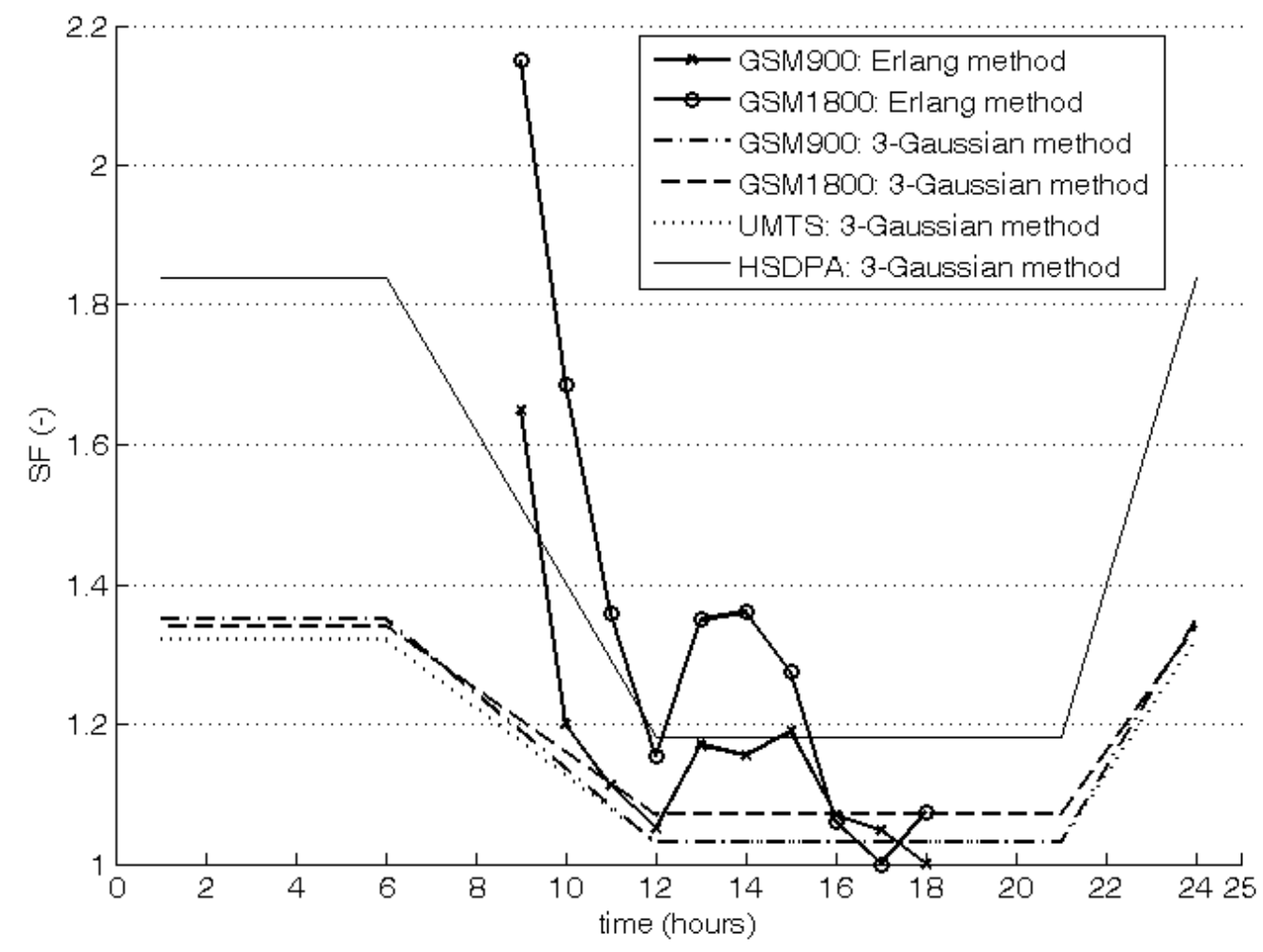

Figure 3 


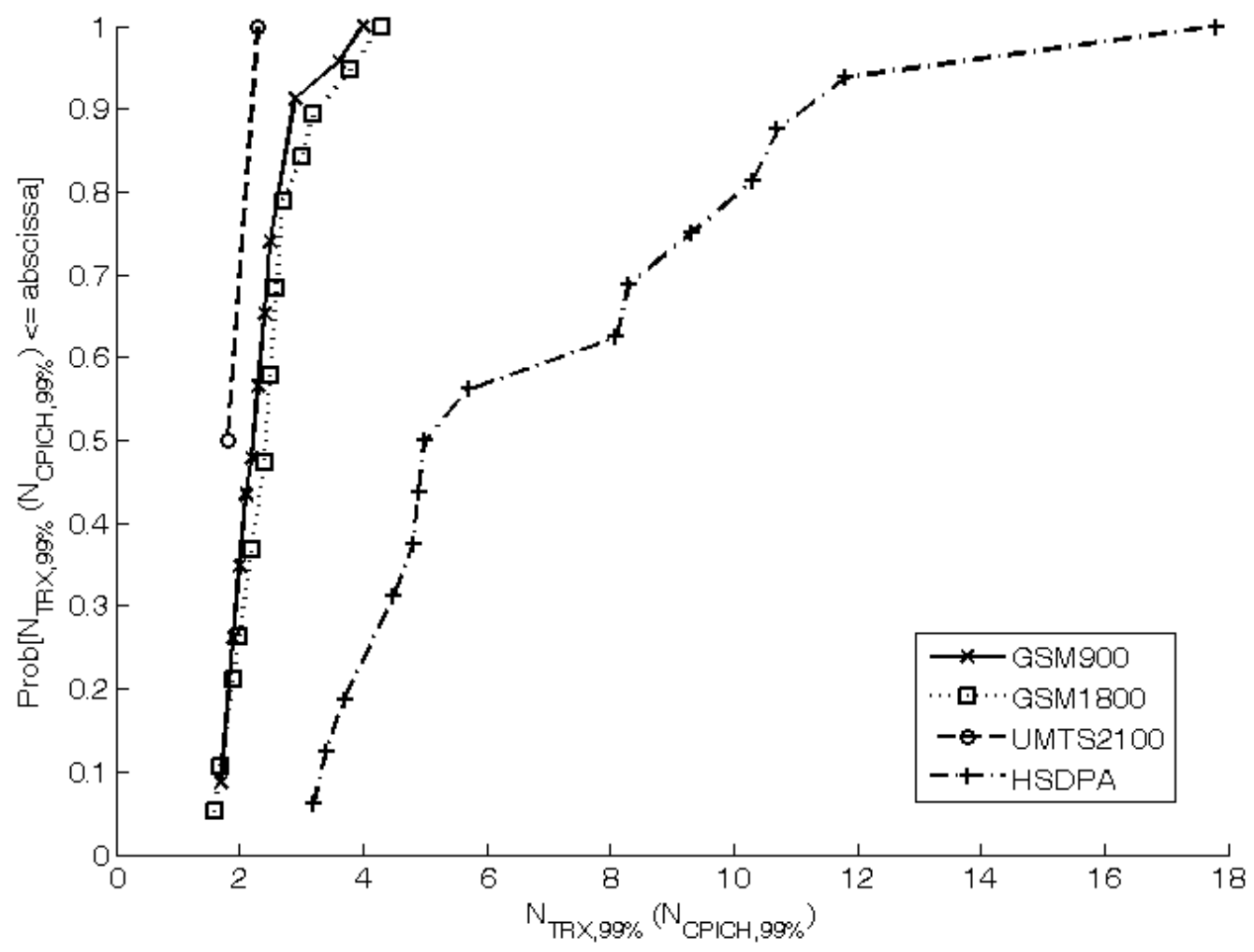

Figure 4 


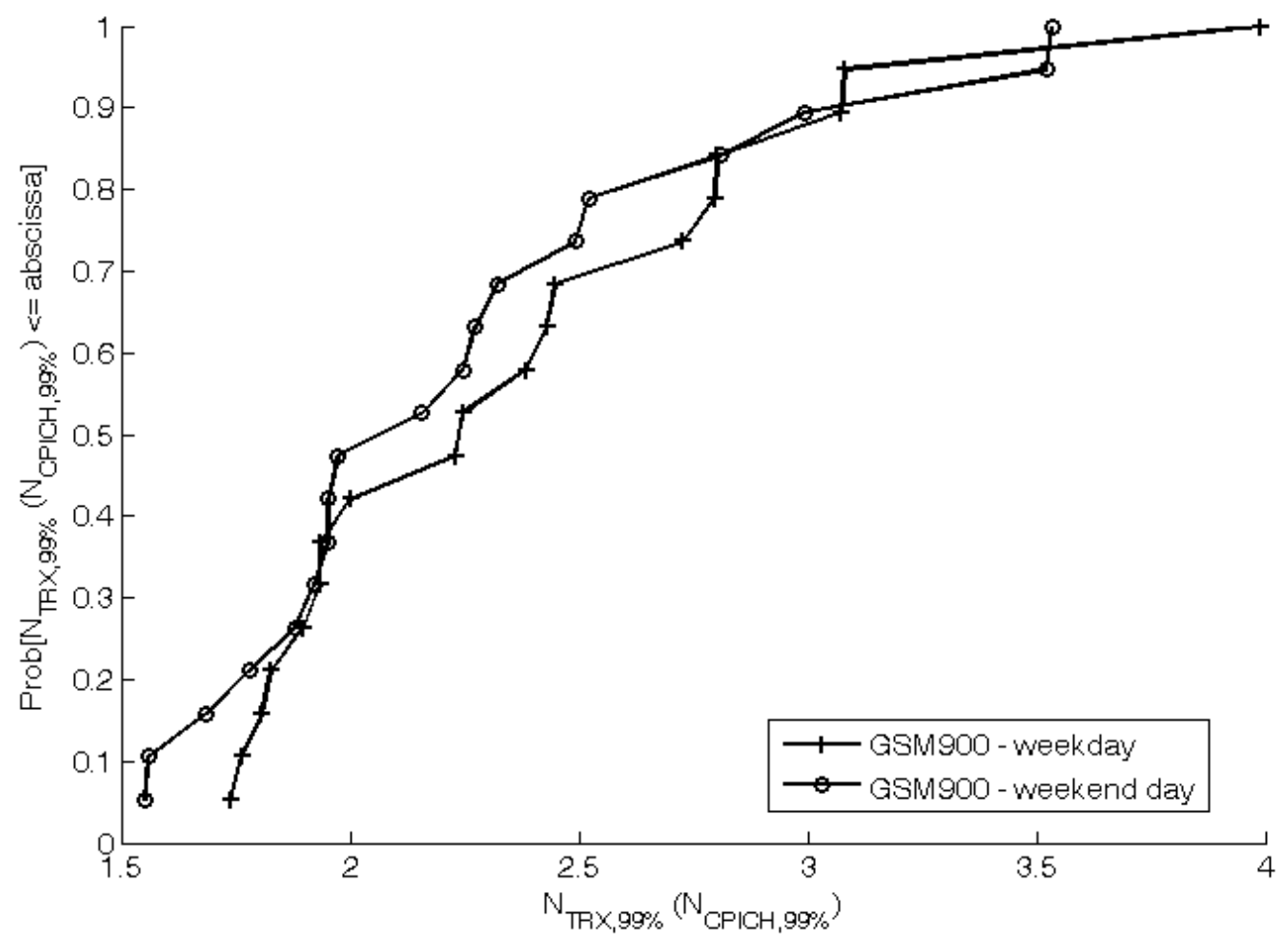

Figure 5 